\title{
A Fractional Epidemic Model with Mittag-Leffler Kernel for COVID-19
}

\author{
Hassan Aghdaoui*1, Mouhcine Tilioua ${ }^{\dagger 1}$, Kottakkaran Sooppy Nisar ${ }^{\ddagger 2}$, \\ Ilyas Khan ${ }^{\S 3}$ \\ ${ }^{1}$ MAMCS Group, FST Errachidia, Moulay Ismail University of Meknes, P.O. Box 509 \\ Boutalamine, 52000, Errachidia, Morocco \\ ${ }^{2}$ Department of Mathematics, College of Arts and Science, Prince Sattam bin Abdulaziz \\ University, Wadi Aldawaser, 11991, Saudi Arabia \\ ${ }^{3}$ Department of Mathematics, College of Science Al-Zulfi, Majmaah University, Al-Majmaah \\ 11952, Saudi Arabia
}

\begin{abstract}
The aim is to explore a COVID-19 SEIR model involving Atangana-Baleanu Caputo type (ABC) fractional derivatives. Existence, uniqueness, positivity, and boundedness of the solutions for the alternative model are established. Some stability results of the proposed system are also presented. Numerical simulations results obtained in this paper, according to the real data, show that the model is more suitable for the disease evolution.
\end{abstract}

Key words: epidemic model, COVID-19, incidence rate, equilibrium points, ABC fractional derivative, existence and uniqueness, numerical simulations.

\section{INTRODUCTION}

The world is currently experiencing an epidemic of infectious disease called COVID-19 []ㅡ, resulting from a new virus belonging to the coronavirus family: SARS-CoV-2. Coronaviruses are a family of various viruses that can infect both humans and animals. Human coronaviruses mainly cause respiratory infections, ranging from mild colds to severe lung disease. They can also be accompanied by digestive disorders such as gastroenteritis. To contain this pandemic which is probably only in its infancy, and avoid a collapse of the health systems, governments must adopt immediate and effective measures in a context of major uncertainty.

The COVID-19 pandemic is drawing attention more than ever to quantitative mathematical modeling. Policy makers and the general public are turning to science, and modeling in particular to gain insight into the complex dynamics of the epidemic, from a local and global perspective, as well as to predict the consequences of possible interventions on the number of cases, hospitalizations and deaths.

Mathematical modeling in epidemiology began in 1760, with Bernoulli's work to assess the effectiveness of vaccination against smallpox. For COVID-19 pandemic, epidemiologists are developing, testing and adjusting models to simulate the spread of this infectious disease; it's about better understanding it and optimizing interventions to control it.

Current models of COVID-19 are generally derived from classic approach for epidemic modeling developed in 1927 by Kermack and McKendrik [25]. The approach divide the population into 3 classes, the number of which changes as a function of time:

\footnotetext{
*aghdaoui.hassan@gmail.com

†m.tilioua@fste.umi.ac.ma

†n.sooppy@psau.edu.sa

§..said@mu.edu.sa
} 
- $S$ individuals: they are the non-carriers of the virus, therefore susceptible to being infected.

- I individuals: they are the infected individuals, whatever the degree of severity of the disease (asymptomatic, symptomatic carriers, etc.).

- $R$ individuals: these are the individuals who have been infected and are recovered. They are therefore immune, for a period of several months, it is believed, for COVID-19.

For COVID-19 disease, it would be suitable to add a population of exposed individuals $(E)$ to the model. Adding more compartments allows for a refined description of specific epidemics with assumptions on transfer from one class to another, see for example $[15,14, \underline{30}]$.

Vaccinating the population would be an ideal solution against COVID-19. In the absence of an available vaccine, confinement (combined with other social distancing measures) will limit the proportion of susceptible people who become infectious. Simulation models are therefore a crucial tool in the fight against COVID-19, helping to understand the enormous efforts required to fight the coronavirus, slow the spread of the epidemic and, thus, save lives.

The usual integer-order models do not enjoy subsequent memory effects occurring in many biological models. The hereditary, as essential property of many biological processes, is introduced through the $\mathrm{ABC}$ operator. Fractional operators have received increasing interest by several directions in the modeling of biological process. Let us brefly review one of the recent papers, in [34] for exemple, the authors proposed and studied the dynamics behavior of COVID-19, that was by analysed an SEIHDR model based on ABC operator, they showed the transmission dynamics of COVID-19 after they analysed the existence results, see $[\underline{1}, \underline{2}, \underline{13}, \underline{12}$, $\underline{11}, \underline{21}, \underline{8}, \underline{7}, \underline{6}, \underline{18}, \underline{19}, \underline{20}, \underline{16}, \underline{10}, \underline{9}, \underline{23}, \underline{24}, \underline{29}, \underline{31}, \underline{32}, \underline{33}]$ and the references therein.

In the present work, due the great importance of $\mathrm{ABC}$ fractional derivative we aim to promote its application to a SEIR epidemic model for COVID-19 and prove existence and uniqueness of bounded and positive solution by using the fixed point theory. After that, fractional derivative effect on epidemic trend is showed through some numerical simulations.

The paper is organized as follows. We first formulated the mathematical model for COVID-19 transmission with $\mathrm{ABC}$ fractional derivatives. Existence, uniqueness, positivity and boundedness of solutions are then proved. Some stability results of the proposed system are presented and the epidemic trend is discussed by using real data and numerical experiments. Finally, a conclusion is drawn in the last section.

\section{FRACTIONAL MODEL}

Before presenting the fractional model, we first recall some definitions and properties that are needed in the next sections.

Definition 1. [7] Let $f \in H^{1}(a, b), a<b$ and $\zeta \in[0,1]$. The definition of the Atangana-Baleanu derivative in Caputo sense of order $\zeta$ of $f$ is

$$
{ }_{a}^{A B C} D_{t}^{\zeta}(f(t))=\frac{B(\zeta)}{1-\zeta} \int_{a}^{t} f^{\prime}(x) E_{\zeta}\left(-\zeta \frac{(t-x)^{\zeta}}{1-\zeta}\right) d x
$$

where $E_{\zeta}(\cdot)$ is the Mittag-Leffler function while $B(\zeta)$ is a normalization function satisfying $B(0)=B(1)=1$.

Defin ition 2. [7] The associated fractional integral is given as

$$
{ }_{a}^{A B C} I_{t}^{\zeta}(f(t))=\frac{1-\zeta}{B(\zeta)} f(t)+\frac{\zeta}{B(\zeta) \Gamma(\zeta)} \int_{a}^{t} f(v)(t-v)^{\zeta-1} d v
$$

We focus on a SEIR system to depict the prevalent characteristics of COVID-19. We 
introduce the $\mathrm{ABC}$ fractional time derivative and the model equations read

$$
\left\{\begin{aligned}
{ }^{A B C}{ }_{0} D_{t}^{\zeta}(S(t)) & =\Lambda-\beta_{1} I S-\beta_{2} E S-\mu S, \\
{ }^{A B C}{ }_{0} D_{t}^{\zeta}(E(t)) & =\beta_{1} I S+\beta_{2} E S-\left(\gamma_{1}+\mu\right) E, \\
{ }^{A B C_{0}} D_{t}^{\zeta}(I(t)) & =\gamma_{1} E-\left(\gamma_{2}+\mu\right) I, \\
{ }^{A B C}{ }_{0} D_{t}^{\zeta}(R(t)) & =\gamma_{2} I-\mu R .
\end{aligned}\right.
$$

The system (3) is completed with the initial conditions

$$
S(0)=S_{0}, \quad E(0)=E_{0}, \quad I(0)=I_{0}, \quad R(0)=R_{0} .
$$

The SEIR model (3) divides the population in total into four separate compartments. $S(t)$ stands for susceptible population at time $t$; the number of asymptomatic infectious individuals is denoted by $E(t)$; the number of symptomatic and infectious population is shown by $I(t)$; the number of infected population quarantined and expecting recovery is described by $R(t)$. We assume $S(t)+E(t)+I(t)+R(t)=N(t)$, where $\Lambda$ is the recruitment rate and $\mu$ is the natural rate of death, $\beta_{1}$ represents the transmission rate of $S$ to $E$ ( $S$ contacted by $I$ ), $\gamma_{1}$ accounts for transmission rate of $E$ to $I$, while $\gamma_{2}$ denotes the transmission rate of $I$ to $R$. As COVID-19 is also infectious in the incubation period, a coefficient $\beta_{2}$ is involved to parameterize the transmission rate of $S$ to $E$ ( $E$ contacted $S$ ). We assume that all parameters are positive. The flow chart is shown in figure 1 in which the boxes represent the different compartments and the arrows the transition between compartments.

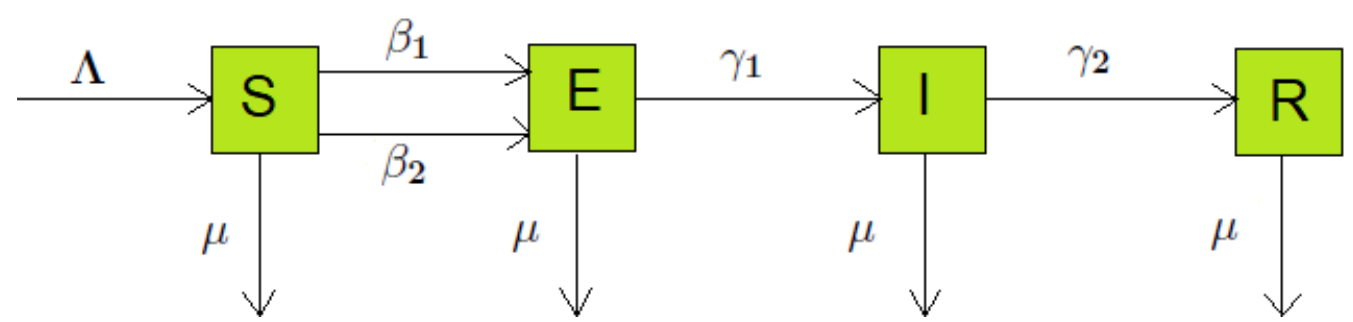

Fig. 1. Flow SEIR model diagram.

\section{EXISTENCE, UNIQUENESS, POSITIVITY AND BOUNDEDNESS}

The fractional order system (3) is complex, non-local and there is no analytical method to solve it. To prove existence of solutions, we make use of a fixed point approach. The system is reformulated as follows:

$$
\left\{\begin{aligned}
{ }^{A B C}{ }_{0} D_{t}^{\zeta}(S(t)) & =\mathcal{H}_{1}(t, S) \\
A B C_{0} D_{t}^{\zeta}(E(t)) & =\mathcal{H}_{2}(t, E) \\
{ }^{A B C_{0}} D_{t}^{\zeta}(I(t)) & =\mathcal{H}_{3}(t, I), \\
{ }_{A B C}{ }_{0} D_{t}^{\zeta}(R(t)) & =\mathcal{H}_{4}(t, R) .
\end{aligned}\right.
$$

Let $\mathcal{E}(N)$ be a Banach space of real continuous functions on $N$. We consider $\mathcal{A}=\mathcal{E}(N) \times$ $\mathcal{E}(N) \times \mathcal{E}(N) \times \mathcal{E}(N)$ equipped with the norm $\|S\|+\|E\|+\|I\|+\|R\|$, where $\|W\|=$ 
$\sup \{|W(t)|, t \in N\}, W=S, E, I, R$.

The ABC fractional order integral allows to convert the system (4) into a Volterra-type integral equation as follows

$$
\left\{\begin{array}{l}
S(t)-S(0)=\frac{1-\zeta}{B(\zeta)} \mathcal{H}_{1}(t, S)+\frac{\zeta}{B(\zeta) \Gamma(\zeta)} \int_{0}^{t}(t-v)^{\zeta-1} \mathcal{H}_{1}(v, S) d v \\
E(t)-E(0)=\frac{1-\zeta}{B(\zeta)} \mathcal{H}_{2}(t, E)+\frac{\zeta}{B(\zeta) \Gamma(\zeta)} \int_{0}^{t}(t-v)^{\zeta-1} \mathcal{H}_{2}(v, E) d v \\
I(t)-I(0)=\frac{1-\zeta}{B(\zeta)} \mathcal{H}_{3}(t, I)+\frac{\zeta}{B(\zeta) \Gamma(\zeta)} \int_{0}^{t}(t-v)^{\zeta-1} \mathcal{H}_{3}(v, I) d v \\
R(t)-R(0)=\frac{1-\zeta}{B(\zeta)} \mathcal{H}_{4}(t, R)+\frac{\zeta}{B(\zeta) \Gamma(\zeta)} \int_{0}^{t}(t-v)^{\zeta-1} \mathcal{H}_{4}(v, R) d v
\end{array}\right.
$$

For the positivity and boundedness of the solutions, we have :

Proposition 1. The solution of system (3) is non-negative and bounded for all $t \geq 0$, provided that initial conditions are non-negative.

Pro of. We have

$$
\begin{aligned}
& { }^{\left.A B C_{0} D_{t}^{\zeta} S\right|_{S=0}}=\Lambda \geq 0, \\
& { }^{\left.A B C_{0} D_{t}^{\zeta} E\right|_{E=0}}=\beta_{1} I S \geq 0, \\
& { }^{\left.A B C_{0} D_{t}^{\zeta} I\right|_{I=0}}=\gamma_{1} E \geq 0, \\
& { }_{\left.A B C_{0} D_{t}^{\zeta} R\right|_{R=0}}=\gamma_{2} I \geq 0 .
\end{aligned}
$$

Therefore, all solutions initiated in $\mathbb{R}_{+}^{4}$ are positive.

For the boundedness, we have

$$
N(t)=S(t)+E(t)+I(t)+R(t)
$$

and

$$
{ }_{0}^{A B C} D_{t}^{\zeta} N(t)=\Lambda-\mu N(t)
$$

So, we deduce that

$$
N(t)=N(0) E_{\zeta}\left(-\mu t^{\zeta}\right)+\frac{\Lambda}{\mu}\left(1-E_{\zeta}\left(-\mu t^{\zeta}\right)\right)
$$

Since $0<E_{\zeta}\left(-\mu t^{\zeta}\right) \leq 1$ and $1-E_{\zeta}\left(-\mu t^{\zeta}\right) \leq 1$, we obtain $N(t) \leq N(0)+\frac{\Lambda}{\mu}$. This achieves the proof.

Theorem 1. Assume that

$$
0 \leq\left(\beta_{1}+\beta_{2}\right) c+\mu<1
$$

then the kernel $\mathcal{H}_{1}$ satisfies the Lipschitz condition and contraction.

Proof. For two functions $S_{1}$ and $S_{2}$, one has

$$
\begin{aligned}
\left\|\mathcal{H}_{1}\left(t, S_{2}\right)-\mathcal{H}_{1}\left(t, S_{1}\right)\right\| & =\left\|-\beta_{1} I S_{2}-\beta_{2} I S_{2}-\mu S_{2}+\beta_{1} I S_{1}+\beta_{1} I S_{1}-\mu S_{1}\right\| \\
& \leq\left\|\beta_{1} I+\beta_{2} E+\mu\right\|\left\|S_{2}(t)-S_{1}(t)\right\| \leq \bar{\delta}_{1}\left\|S_{2}(t)-S_{1}(t)\right\| .
\end{aligned}
$$


where $\bar{\delta}_{1}=\left(\beta_{1}+\beta_{2}\right) c+\mu, c=\max \left(b, c^{\prime}\right)$ and $\|E\| \leq b,\|I\| \leq c^{\prime}$. We conclude that the Lipschitz condition is satisfied for $\mathcal{H}_{1}$.

Moreover, if $0 \leq\left(\beta_{1}+\beta_{2}\right) c+\mu<1$, then there follows a contraction.

The remaining kernels can be easily handled to get

$$
\begin{gathered}
\left\|\mathcal{H}_{2}\left(t, E_{2}\right)-\mathcal{H}_{2}\left(t, E_{1}\right)\right\| \leq \bar{\delta}_{2}\left\|E_{2}(t)-E_{1}(t)\right\| . \\
\left\|\mathcal{H}_{3}\left(t, I_{2}\right)-\mathcal{H}_{3}\left(t, I_{1}\right)\right\| \leq \bar{\delta}_{3}\left\|I_{2}(t)-I_{1}(t)\right\| . \\
\left\|\mathcal{H}_{4}\left(t, R_{2}\right)-\mathcal{H}_{4}\left(t, R_{1}\right)\right\| \leq \bar{\delta}_{4}\left\|R_{2}(t)-R_{1}(t)\right\| .
\end{gathered}
$$

The same procedure discussed for example in [26] can be applied to get existence and uniqueness for the fractional model (3). In particular, we have

Theorem 2. Assume that

$$
\frac{1-\zeta}{B(\zeta)} \bar{\delta}_{i}+\frac{t_{\max }^{\zeta}}{B(\zeta) \Gamma(\zeta)} \bar{\delta}_{i}<1, i=1,2,3,4
$$

for some $t_{\text {max }}^{\zeta}$, then the fractional model (3) admits a unique solution.

\section{STABILITY RESULTS}

We can see that the closed set

$$
\Gamma=\left\{(S, E, I, R) \in \mathbb{R}_{+}^{4}: S+E+I+R \leq \frac{\Lambda}{\mu}\right\}
$$

is a positively invariant set for the system (3). In fact, from (6), we can conclude that $N(t) \leq \frac{\Lambda}{\mu}$ as $t \longrightarrow \infty$. Hence, for any initial condition in $\Gamma$, the solution of fractional model (3) does not leave $\Gamma$ for all $t \geq 0$ and the system is well posed for mathematical and epidemiological considerations in $\Gamma$. In this section, we shall prove some stability result for the model (3) with integer-order derivative, namely

$$
\left\{\begin{array}{l}
\dot{S}(t)=\Lambda-\beta_{1} I S-\beta_{2} E S-\mu S \\
\dot{E}(t)=\beta_{1} I S+\beta_{2} E S-\left(\gamma_{1}+\mu\right) E \\
\dot{I}(t)=\gamma_{1} E-\left(\gamma_{2}+\mu\right) I \\
\dot{R}(t)=\gamma_{2} I-\mu R
\end{array}\right.
$$

Let us first calculate the basic reproduction number $\mathcal{R}_{0}$ and the endemic equilibrium.

\section{Equilibria and basic reproduction number}

The disease-free equilibrium is obtained by letting $I=0$ in $(\underline{12})$, we have the disease-free equilibrium state

$$
X_{0}=\left(\tilde{S}_{0}, 0,0,0\right)=\left(\frac{\Lambda}{\mu}, 0,0,0\right)
$$


The infectious part of the system $(\underline{12)}$ is

$$
\left\{\begin{array}{l}
\dot{E}(t)=\beta_{1} I S+\beta_{2} E S-\left(\gamma_{1}+\mu\right) E, \\
\dot{I}(t)=\gamma_{1} E-\left(\gamma_{2}+\mu\right) I .
\end{array}\right.
$$

By linearized sytem at the point $X_{0}$, we have the following corresponding matrix :

$$
A=\left(\begin{array}{cc}
\beta_{2} \tilde{S}_{0}-\gamma_{1}-\mu & \beta_{1} \tilde{S}_{0} \\
\gamma_{1} & -\gamma_{2}-\mu
\end{array}\right) .
$$

The matrix $A$ can be rewritten as $A=\mathcal{V}-\mathcal{S}$ where

$$
\mathcal{V}=\left(\begin{array}{cc}
\beta_{2} & \beta_{1} \\
0 & 0
\end{array}\right), \mathcal{S}=\left(\begin{array}{cc}
\gamma_{1}+\mu & 0 \\
-\gamma_{1} & \gamma_{2}+\mu
\end{array}\right)
$$

The next generation matrix [4] is :

$$
\mathcal{V} \mathcal{S}^{-1}=\left(\begin{array}{cc}
\frac{\beta_{2} \tilde{S}_{0}\left(\gamma_{2}+\mu\right)+\gamma_{1} \beta_{1} \tilde{S}_{0}}{\left(\gamma_{1}+\mu\right)\left(\gamma_{2}+\mu\right)} & \frac{\beta_{1} \tilde{S}_{0}}{\gamma_{2}+\mu} \\
\frac{\gamma_{1}}{\gamma_{1}+\mu} & 0
\end{array}\right) .
$$

So, the basic reproduction number is

$$
\mathcal{R}_{0}=\frac{\beta_{2}\left(\gamma_{2}+\mu\right)+\gamma_{1} \beta_{1}}{\left(\gamma_{1}+\mu\right)\left(\gamma_{2}+\mu\right)} \tilde{S}_{0}
$$

In the next, we prove that model $\underline{(12)}$ has a unique endemic equilibrium.

L e m m a 1. If $\mathcal{R}_{0}>1$, then the system (12) admits a unique endemic equilibrium $X^{*}$, and no endemic if $\mathcal{R}_{0} \leq 1$.

Pro of. In order to determine the endemic equilibrium, we set the right-hand side of $(12)$ to zero. We find that

$$
\begin{gathered}
\Lambda-\beta_{1} I(t) S(t)-\beta_{2} E(t) S(t)-\mu S(t)=0 \\
\beta_{1} I(t) S(t)+\beta_{2} E(t) S(t)-\left(\gamma_{1}+\mu\right) E(t)=0 \\
\gamma_{1} E(t)-\left(\gamma_{2}+\mu\right) I(t)=0 \\
\gamma_{2} I(t)-\mu R(t)=0 .
\end{gathered}
$$

By adding $\underline{(14)}$ and $(\underline{15})$, we get

$$
E(t)=\frac{1}{\left(\gamma_{1}+\mu\right)}(\Lambda-\mu S(t))
$$

From $\underline{(16)}$ we obtain

$$
I(t)=\frac{\gamma_{1}}{\gamma_{2}+\mu} E(t)
$$


while from $(17)$ it follows that

$$
R(t)=\frac{\gamma_{2}}{\mu} I(t)
$$

Using $\underline{(19)}$ in $(\underline{15)}$ one gets

$$
E(t)\left[S(t)\left(\frac{\beta_{1} \gamma_{1}}{\gamma_{2}+\mu}+\beta_{2}\right)-\left(\gamma_{1}+\mu\right)\right]=0
$$

That is, or $E(t)=0$ or $S(t)=\frac{\left(\gamma_{1}+\mu\right)\left(\gamma_{2}+\mu\right)}{\beta_{1} \gamma_{1}+\beta_{2}\left(\gamma_{2}+\mu\right)}$. If $E(t)=0$, then from (18) we obtain $S(t)=\frac{\Lambda}{\mu}$, while from (19) $I(t)=0$, which implies by (20) $R(t)=0$. So the endemic equilibrium state $X_{*}=\left(S_{*}, E_{*}, I_{*}, R_{*}\right)$ with

$$
\left\{\begin{array}{l}
S_{*}=\frac{\left(\gamma_{1}+\mu\right)\left(\gamma_{2}+\mu\right)}{\beta_{1} \gamma_{1}+\beta_{2}\left(\gamma_{2}+\mu\right)}=\frac{\tilde{S}_{0}}{\mathcal{R}_{0}}, \\
E_{*}=\frac{1}{\left(\gamma_{1}+\mu\right)}\left(\Lambda-\mu S_{*}\right)=\frac{\Lambda}{\mathcal{R}_{0}\left(\gamma_{1}+\mu\right)}\left(\mathcal{R}_{0}-1\right)>0, \\
I_{*}=\frac{\gamma_{1}}{\gamma_{2}+\mu} E_{*}, \\
R_{*}=\frac{\gamma_{2}}{\mu} I_{*} .
\end{array}\right.
$$

\section{Stability}

For local stability of the disease-free equilibrium state, we have

Theorem 3. If $\mathcal{R}_{0}<1$, then the disease-free equilibrium state $X_{0}$ of system (12) is locally asymptotically stable.

Pro of. We evaluate the Jacobian matrix of system $\left(\underline{12)}\right.$ at $X_{0}$ to get

$$
J\left(X_{0}\right)=\left(\begin{array}{cccc}
-\mu & -\beta_{2} \tilde{S}_{0} & -\beta_{1} \tilde{S}_{0} & 0 \\
0 & \beta_{2} \tilde{S}_{0}-\left(\gamma_{1}+\mu\right) & \beta_{1} \tilde{S}_{0} & 0 \\
0 & \gamma_{1} & -\left(\gamma_{2}+\mu\right) & 0 \\
0 & 0 & \gamma_{2} & -\mu
\end{array}\right),
$$

and the characteristic polynomial reads

$$
\begin{aligned}
P(X) & =(X+\mu)\left(X^{2}+\left(\gamma_{1}+\gamma_{2}+2 \mu-\beta_{2} \tilde{S}_{0}\right) X+\left(\gamma_{1}+\mu-\beta_{2} \tilde{S}_{0}\right)\left(\gamma_{2}+\mu\right)-\beta_{1} \gamma_{1} \tilde{S}_{0}\right) \\
& =(X+\mu)\left(X^{2}+\left(\gamma_{1}+\gamma_{2}+2 \mu-\beta_{2} \tilde{S}_{0}\right) X+\left(\gamma_{1}+\mu\right)\left(\gamma_{2}+\mu\right)\left(1-\mathcal{R}_{0}\right) .\right.
\end{aligned}
$$


Polynomial $\underline{(22)}$ has a real roots which are

$$
\begin{aligned}
X_{1} & =-\mu<0 \\
X_{2}+X_{3} & =-\left(\gamma_{1}+\gamma_{2}+2 \mu-\beta_{2} \tilde{S}_{0}\right)<0, \\
X_{2} X_{3} & =\left(\gamma_{1}+\mu\right)\left(\gamma_{2}+\mu\right)\left(1-\mathcal{R}_{0}\right)>0 .
\end{aligned}
$$

So $X_{2}<0$ and $X_{3}<0$. Therefore, by applying the Routh-Hurwitz's criterion, the disease-free equilibrium $X_{0}$ is locally asymptotically stable. For the global stability of the disease free equilibrium state, we have

Th e o r e m 4. The disease-free equilibrium state is globally asymptotically stable if $\mathcal{R}_{0}<1$.

P r o of. We define the following Lyapunov function $V$ by

$$
V(S, E, I)=\frac{1}{\mu+\gamma_{2}}\left[\frac{\beta_{2}\left(\mu+\gamma_{2}\right)+\beta_{1} \gamma_{1}}{\mu+\gamma_{1}} E+\beta_{1} I\right]
$$

We have

$$
\begin{aligned}
\dot{V} & =\frac{1}{\mu+\gamma_{2}}\left[\frac{\beta_{2}\left(\mu+\gamma_{2}\right)+\beta_{1} \gamma_{1}}{\mu+\gamma_{1}} \dot{E}+\beta_{1} \dot{I}\right] \\
& =\frac{1}{\mu+\gamma_{2}}\left[\frac{\beta_{2}\left(\mu+\gamma_{2}\right)+\beta_{1} \gamma_{1}}{\mu+\gamma_{1}}\left(\beta_{1} S I+\beta_{2} S E-\left(\gamma_{1}+\mu\right) E\right)+\beta_{1}\left(\gamma_{1} E-\left(\gamma_{2}+\mu\right) I\right)\right] \\
& =\frac{\mathcal{R}_{0}}{\tilde{S}_{0}}\left(\beta_{1} S I+\beta_{2} S E-\left(\gamma_{1}+\mu\right) E\right)+\frac{\beta_{1} \gamma_{1}}{\mu+\gamma_{2}} E-\beta_{1} I \\
& =\frac{\mathcal{R}_{0}}{\tilde{S}_{0}} \beta_{1} S I+\frac{\mathcal{R}_{0}}{\tilde{S}_{0}} \beta_{2} S E-\frac{\mathcal{R}_{0}}{\tilde{S}_{0}}\left(\gamma_{1}+\mu\right) E+\frac{\beta_{1} \gamma_{1}}{\mu+\gamma_{2}} E-\beta_{1} I \\
& =\beta_{1} I\left(\frac{\mathcal{R}_{0}}{\tilde{S}_{0}} S-1\right)+\left(\frac{\mathcal{R}_{0}}{\tilde{S}_{0}} \beta_{2} S-\frac{\mathcal{R}_{0}}{\tilde{S}_{0}}\left(\gamma_{1}+\mu\right)+\frac{\beta_{1} \gamma_{1}}{\mu+\gamma_{2}}\right) E
\end{aligned}
$$

Then

$$
\begin{aligned}
\dot{V} & =\beta_{1} I\left(\frac{\mathcal{R}_{0}}{\tilde{S}_{0}} S-1\right)+\left(\frac{\mathcal{R}_{0}}{\tilde{S}_{0}} \beta_{2} S-\frac{\beta_{2}\left(\mu+\gamma_{2}\right)+\beta_{1} \gamma_{1}}{\left(\mu+\gamma_{1}\right)\left(\mu+\gamma_{2}\right)}\left(\gamma_{1}+\mu\right)+\frac{\beta_{1} \gamma_{1}}{\mu+\gamma_{2}}\right) E \\
& =\beta_{1} I\left(\frac{\mathcal{R}_{0}}{\tilde{S}_{0}} S-1\right)+\left(\frac{\mathcal{R}_{0}}{\tilde{S}_{0}} \beta_{2} S-\beta_{2}\right) E \\
& =\left(\beta_{1} I+\beta_{2} E\right)\left(\frac{\mathcal{R}_{0}}{\tilde{S}_{0}} S-1\right) \\
& \leq\left(\beta_{1} I+\beta_{2} E\right)\left(\frac{S}{\tilde{S}_{0}}-1\right) \leq 0
\end{aligned}
$$

Hence $\dot{V} \leq 0$, if $\mathcal{R}_{0}<1$. We deduce that the disease-free equilibrium state is globally 
asymptotically stable.

Th e or e m 5. The unique endemic equilibrium is globally asymptotically stable if $\mathcal{R}_{0}>1$.

Pro of. We consider the Lyapunov function $L=L(S, E, I)$ for $S, E, I>0$, such that

$$
L=\int_{S_{*}}^{S} \frac{x-S_{*}}{x} d x+\int_{E_{*}}^{E} \frac{x-E_{*}}{x} d x+\int_{I_{*}}^{I} \frac{x-I_{*}}{x} d x .
$$

Clearly $L=L(S, E, I) \geq 0$, for $S, E, I>0$. By using the endemic equilibrium state $X_{*}=$ $\left(S_{*}, E_{*}, I_{*}, R_{*}\right)$, we have

$$
\begin{aligned}
\dot{L} & =\frac{S-S_{*}}{S} \dot{S}(t)+\frac{E-E_{*}}{E} \dot{E}(t)+\frac{I-I_{*}}{I} \dot{I}(t) \\
& =\frac{S-S_{*}}{S}\left(\beta_{1} I_{*} S_{*}\left(1-\frac{S I}{S_{*} I_{*}}\right)+\beta_{2} E_{*} S_{*}\left(1-\frac{E S}{E_{*} S_{*}}\right)+\mu S_{*}\left(1-\frac{S}{S_{*}}\right)\right) \\
& +\frac{E-E_{*}}{E}\left(\beta_{1} I_{*} S_{*}\left(\frac{S I}{S_{*} I_{*}}-\frac{E}{E_{*}}\right)+\beta_{2} E_{*} S_{*}\left(\frac{E S}{E_{*} S_{*}}-\frac{E}{E_{*}}\right)\right) \\
& +\frac{I-I_{*}}{I}\left(\gamma_{1} E_{*}\left(\frac{E}{E_{*}}-\frac{I}{I_{*}}\right)\right) \\
& =\beta_{1} I_{*} S_{*}\left(2+\frac{I}{I_{*}}-\frac{S}{S_{*}}-\frac{E}{E_{*}}-\frac{S I E_{*}}{S_{*} I_{*} E}\right)+\left(\beta_{2} E_{*} S_{*}+\mu S_{*}\right)\left(2-\frac{S_{*}}{S}-\frac{S}{S_{*}}\right) \\
& +\gamma_{1} E_{*}\left(1+\frac{E}{E_{*}}-\frac{I}{I_{*}}-\frac{I_{*} E}{I E_{*}}\right) .
\end{aligned}
$$

Note that we have

$$
2-\frac{S}{S_{*}}-\frac{S_{*}}{S} \leq 0
$$

and if

$$
\begin{aligned}
2+\frac{I}{I_{*}}-\frac{S}{S_{*}}-\frac{E}{E_{*}}-\frac{S I E_{*}}{S_{*} I_{*} E} & \leq 0 \\
1+\frac{E}{E_{*}}-\frac{I}{I_{*}}-\frac{I_{*} E}{I E_{*}} & \leq 0
\end{aligned}
$$

then, by Lyapunov stability theorem, it ensures that the model is globally asymptotically stable at $X_{*}$ when $\mathcal{R}_{0}>1$.

\section{NUMERICAL SIMULATIONS AND DISCUSSION}

Numerical simulations for solving the ABC fractional model (3) are based on formulas (5). In fact, we use the two points Lagrange interpolation polynomial to approximate the fractional integral [27]. We first present the method briefly and than apply it to obtain an iterative scheme. By applying the fundamental theorem of fractional calculus we convert the system (4) into a Volterra-type integral equation (5).

At $t=t_{n+1}, n=0,1,2 \ldots$, we have 


$$
S\left(t_{n+1}\right)-S(0)=\frac{1-\zeta}{B(\zeta)} \mathcal{H}_{1}\left(t_{n}, S\right)+\frac{\zeta}{B(\zeta) \Gamma(\zeta)} \int_{0}^{t_{n+1}}\left(t_{n+1}-v\right)^{\zeta-1} \mathcal{H}_{1}(v, S) d v
$$

So

$$
\begin{aligned}
S\left(t_{n+1}\right)= & S(0)+\frac{1-\zeta}{B(\zeta)} \mathcal{H}_{1}\left(t_{n}, S\right)+\frac{\zeta}{B(\zeta) \Gamma(\zeta)} \sum_{k=0}^{n} \int_{t_{k}}^{t_{k+1}}\left(t_{n+1}-v\right)^{\zeta-1} \mathcal{H}_{1}(v, S) d v \\
= & \frac{1-\zeta}{B(\zeta)} \mathcal{H}_{1}\left(t_{n}, S\right)+\frac{\zeta}{B(\zeta) \Gamma(\zeta)} \sum_{k=0}^{n}\left[\frac{\mathcal{H}_{1}\left(t_{k}, S\left(t_{k}\right)\right)}{h} \int_{t_{k}}^{t_{k+1}}\left(v-t_{k-1}\right)\left(t_{n+1}-v\right)^{\zeta-1} d v\right. \\
- & \left.\frac{\mathcal{H}_{1}\left(t_{k-1}, S\left(t_{k-1}\right)\right)}{h} \int_{t_{k}}^{t_{k+1}}\left(v-t_{k}\right)\left(t_{n+1}-v\right)^{\zeta-1} d v\right]+S\left(t_{0}\right) \\
= & S\left(t_{0}\right)+\frac{1-\zeta}{B(\zeta)} \mathcal{H}_{1}\left(t_{n}, S\left(t_{n}\right)\right)+\frac{\zeta}{B(\zeta)} \times \\
& \sum_{k=0}^{n}\left[\frac{h^{\zeta} \mathcal{H}_{1}\left(t_{k}, S\left(t_{k}\right)\right)}{\Gamma(\zeta+2)}\left((n+1-k)^{\zeta}(n-k+2+\zeta)-(n-k)^{\zeta}(n-k+2+2 \zeta)\right)\right. \\
- & \left.\frac{h^{\zeta} \mathcal{H}_{1}\left(t_{k-1}, S\left(t_{k-1}\right)\right)}{\Gamma(\zeta+2)}\left((n+1-k)^{\zeta+1}-(n-k)^{\zeta}(n-k+1+\zeta)\right)\right]
\end{aligned}
$$

In a similar way, we get formulas for $E, I$ and $R$. 
The numerical algorithm reads

- $\quad S\left(t_{n+1}\right)=S\left(t_{0}\right)+\frac{1-\zeta}{B(\zeta)} \mathcal{H}_{1}\left(t_{n}, S\left(t_{n}\right)\right)+\frac{\zeta}{B(\zeta)} \times$

$$
\begin{aligned}
& \sum_{k=0}^{n}\left[\frac{h^{\zeta} \mathcal{H}_{1}\left(t_{k}, S\left(t_{k}\right)\right)}{\Gamma(\zeta+2)}\left((n+1-k)^{\zeta}(n-k+2+\zeta)-(n-k)^{\zeta}(n-k+2+2 \zeta)\right)\right. \\
& \left.-\frac{h^{\zeta} \mathcal{H}_{1}\left(t_{k-1}, S\left(t_{k-1}\right)\right)}{\Gamma(\zeta+2)}\left((n+1-k)^{\zeta+1}-(n-k)^{\zeta}(n-k+1+\zeta)\right)\right]
\end{aligned}
$$

- $\quad E\left(t_{n+1}\right)=E\left(t_{0}\right)+\frac{1-\zeta}{B(\zeta)} \mathcal{H}_{2}\left(t_{n}, E\left(t_{n}\right)\right)+\frac{\zeta}{B(\zeta)} \times$

$$
\begin{aligned}
& \sum_{k=0}^{n}\left[\frac{h^{\zeta} \mathcal{H}_{2}\left(t_{k}, E\left(t_{k}\right)\right)}{\Gamma(\zeta+2)}\left((n+1-k)^{\zeta}(n-k+2+\zeta)-(n-k)^{\zeta}(n-k+2+2 \zeta)\right)\right. \\
& \left.-\frac{h^{\zeta} \mathcal{H}_{2}\left(t_{k-1}, E\left(t_{k-1}\right)\right)}{\Gamma(\zeta+2)}\left((n+1-k)^{\zeta+1}-(n-k)^{\zeta}(n-k+1+\zeta)\right)\right]
\end{aligned}
$$

- $\quad I\left(t_{n+1}\right)=I\left(t_{0}\right)+\frac{1-\zeta}{B(\zeta)} \mathcal{H}_{3}\left(t_{n}, I\left(t_{n}\right)\right)+\frac{\zeta}{B(\zeta)} \times$

$$
\sum_{k=0}^{n}\left[\frac{h^{\zeta} \mathcal{H}_{3}\left(t_{k}, I\left(t_{k}\right)\right)}{\Gamma(\zeta+2)}\left((n+1-k)^{\zeta}(n-k+2+\zeta)-(n-k)^{\zeta}(n-k+2+2 \zeta)\right)\right.
$$

$$
\left.-\frac{h^{\zeta} \mathcal{H}_{3}\left(t_{k-1}, I\left(t_{k-1}\right)\right)}{\Gamma(\zeta+2)}\left((n+1-k)^{\zeta+1}-(n-k)^{\zeta}(n-k+1+\zeta)\right)\right]
$$

- $R\left(t_{n+1}\right)=R\left(t_{0}\right)+\frac{1-\zeta}{B(\zeta)} \mathcal{H}_{4}\left(t_{n}, R\left(t_{n}\right)\right)+\frac{\zeta}{B(\zeta)} \times$

$$
\begin{aligned}
& \sum_{k=0}^{n}\left[\frac{h^{\zeta} \mathcal{H}_{4}\left(t_{k}, R\left(t_{k}\right)\right)}{\Gamma(\zeta+2)}\left((n+1-k)^{\zeta}(n-k+2+\zeta)-(n-k)^{\zeta}(n-k+2+2 \zeta)\right)\right. \\
& \left.-\frac{h^{\zeta} \mathcal{H}_{4}\left(t_{k-1}, R\left(t_{k-1}\right)\right)}{\Gamma(\zeta+2)}\left((n+1-k)^{\zeta+1}-(n-k)^{\zeta}(n-k+1+\zeta)\right)\right]
\end{aligned}
$$

for $n=0,1, \ldots$

The stability and convergence results for the above scheme can be obtained by using the algorithm presented in [28].

In order to apply the fractional model (3) to simulate the COVID-19 epidemic in Morocco, we consider the reported data from March 02, 2020, till June 17, 2020 and we make use of the data from [22]. Estimation of the parameters $\beta_{1}, \beta_{2}, \gamma_{1}$ and $\gamma_{2}$ can be obtained by [ $\left.\underline{3}\right]$

$$
\beta_{1}=\frac{\mathcal{R}_{0}}{\mathrm{TI}}, \beta_{2}=\frac{\mathcal{R}_{0}}{\mathrm{TE}}, \gamma_{1}=\frac{1}{\mathrm{TE}}, \gamma_{2}=\frac{1}{\mathrm{TI}}
$$


where TI and TE are respectively the infectious and incubation periods. We precise that incubation period is the time elapsed between exposure and symptoms manifestation. The basic reproduction number $\mathcal{R}_{0} \simeq 1.01$ (May 5th, [22]). The $\mathcal{R}_{0}$ value shows that the quarantine strategy and ensuing measures have a significant role in the epidemic trend. So, we can estimate the parameters values and the results are summarized in Table 1.

Table 1. SEIR model parameters

\begin{tabular}{|c|c|c|}
\hline Notation & Description of parameter & Value \\
& & \\
\hline$S_{0}$ & Initial susceptible population & 798 \\
$E_{0}$ & Initial exposed population & 5 \\
$I_{0}$ & Initial infected population & 1 \\
$R_{0}$ & Initial recovered population & 0 \\
$\mu$ & Natural death rate & 0.01 \\
$\Lambda$ & Flux of population & 20 \\
$\beta_{1}$ & Transmission rate of $S$ to $I(I$ contacted $S)$ & 0.072 \\
$\beta_{2}$ & Transmission rate of $S$ to $E(E$ contacted $S)$ & 0.337 \\
$\gamma_{1}$ & Transmission rate of $E$ to $I$ & 0.333 \\
$\gamma_{2}$ & Transmission rate of $I$ to $R$ & 0.0714 \\
TI & Infectious period & 14.00 \\
TE & Incubation period & 3.00 \\
\hline
\end{tabular}

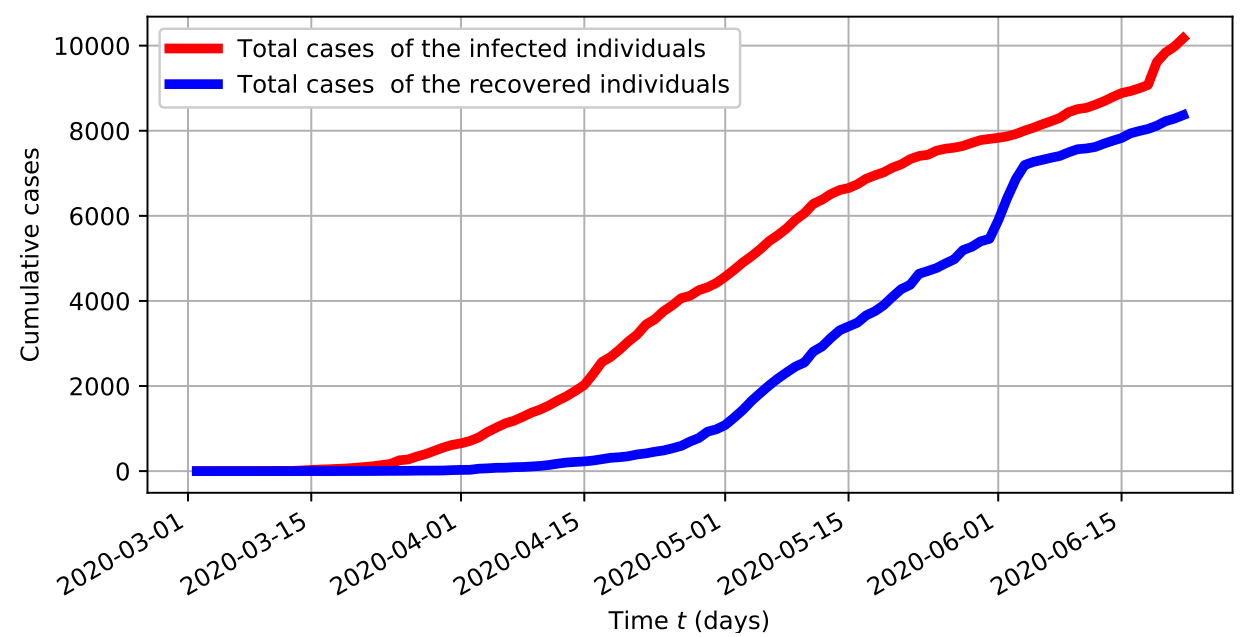

Fig. 2. Cumulative curves of infected and recovered individuals [22] . 
The Figure 2 shows the cumulative cases of the infected and recovered individuals in Morocco from March 2th to June 17th, 2020.

Simulations are carried out up to 120 days and $t=0$ represents March 02, 2020. The Figure $\underline{3}$ shows the evolution of different compartments for $\zeta=0.98$. In Figures $\underline{5}, \underline{6}, \underline{7}$ and $\underline{8}$, the $\bar{b}$ havior of the model components are visualized by varying the fractional order.

We can observe from Figure 4 that, when $\zeta=1$, the results obtained using the ABC derivatives are close to the ones in the classical case. However, as $\zeta$ smaller than one, the obtained results are slightly different.

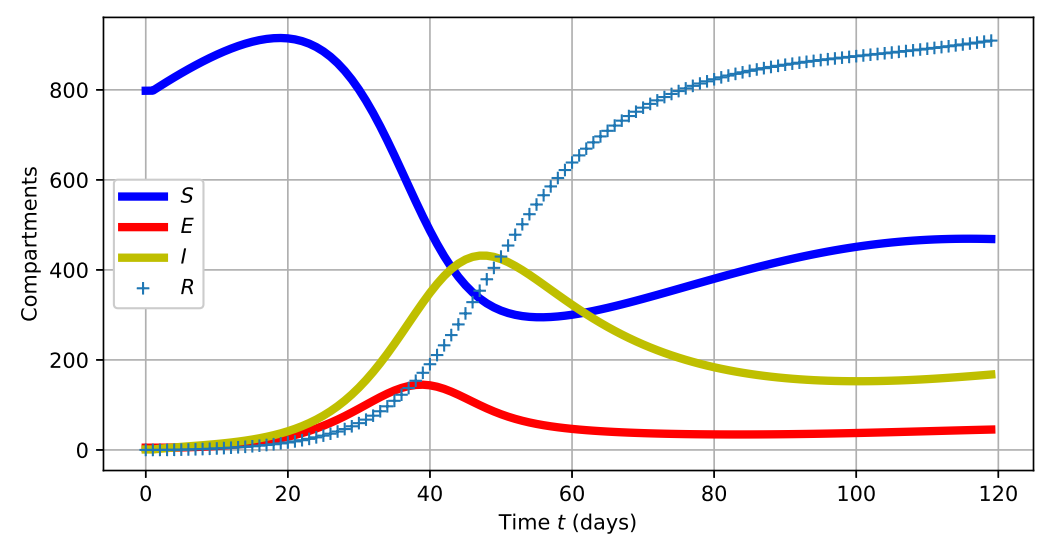

Fig. 3. Numerical simulations for system (3) for $\zeta=0.98$.
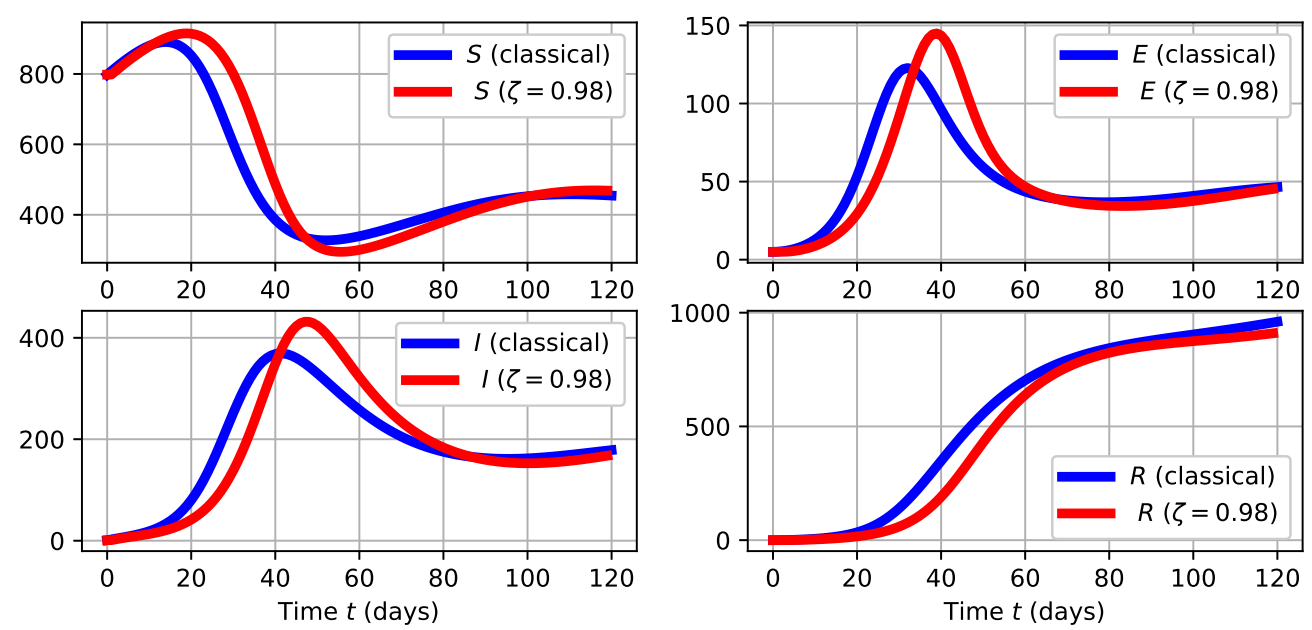

Fig. 4. The comparison between the solutions obtained by using the function odeint to solve the differential equations in Python and the numerical solutions obtained by the proposed method with $\zeta=0.98$.

Next, we present graphical results of the COVID-19 model (3) along with real data. So to make a better illustration, we tested various values of the fractional order $\zeta$. Notice that in the Figures 9 and 10 the solid curves is the model simulations for infected and recovered individuals in fractional case, while the dotted curves represent the real curves from March 2 th to June 17 th, 2020. From these figures we illustrate the effect of fractional order $\zeta$ on both infected and 


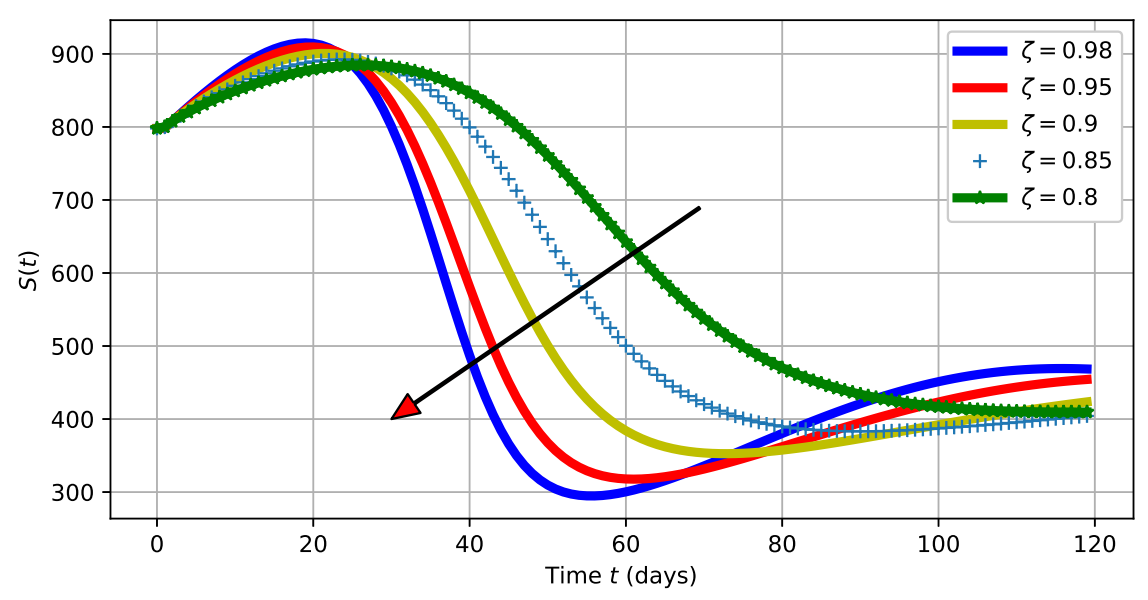

Fig. 5. Evolution of the susceptible compartment for varying fractional order $\zeta$.

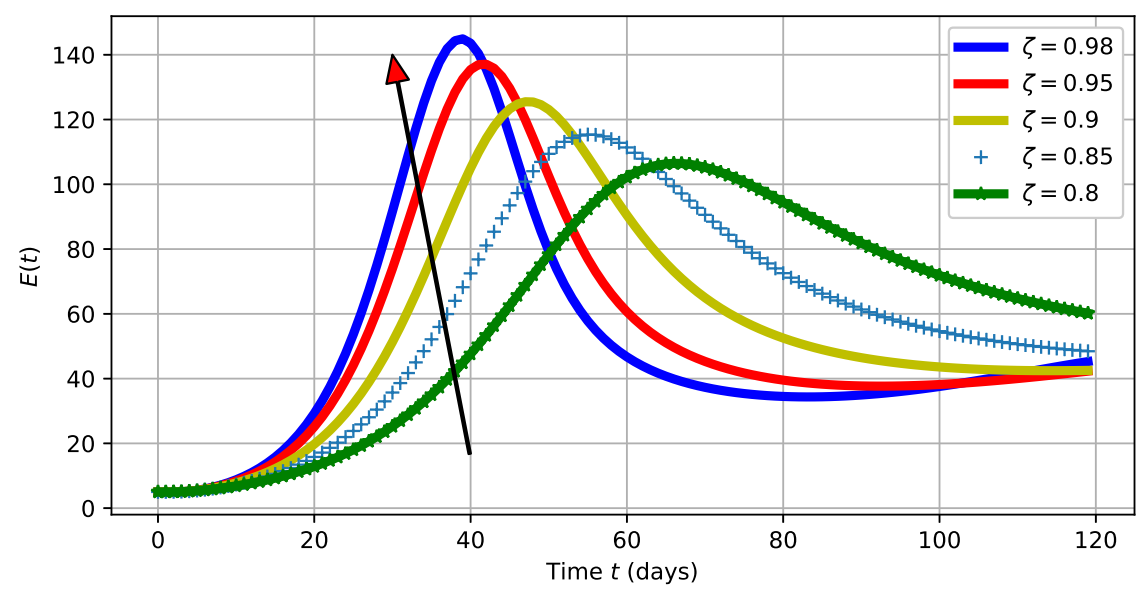

Fig. 6. Evolution of the exposed compartment for varying fractional order $\zeta$.

recovered individuals in case of Morocco. It is important to note that the dynamics of the infected individuals can be simulated for $\zeta \in[0.9,1]$, while the recovered individuals can be simulated for $\zeta<0.9$. These results may be explained by the sudden outbreak of the epidemic, or a considerable number of infected and asymptomatic infectious individuals were not quarantined promptly. So we need to apply an optimal control strategy to control the behavior of the system. The numerical simulations allow to conclude that notable results for the case of Morocco can be obtained for fractional order $\zeta<1$.

\section{CONCLUDING REMARKS}

In this paper, a fractional SEIR model in the ABC fractional derivative sense for COVID-19 modeling is considered. Results on existence, uniqueness, positivity and boundedness of the solutions, are proved. Some stability results are also obtained. The graphical representations show that the fractional model provides a suitable solution than that of the integer case, because it allows to elucidate the correct information of the infection transmission. Models cannot predict what will happen, but they can rather help to understand what might happen so that we can 


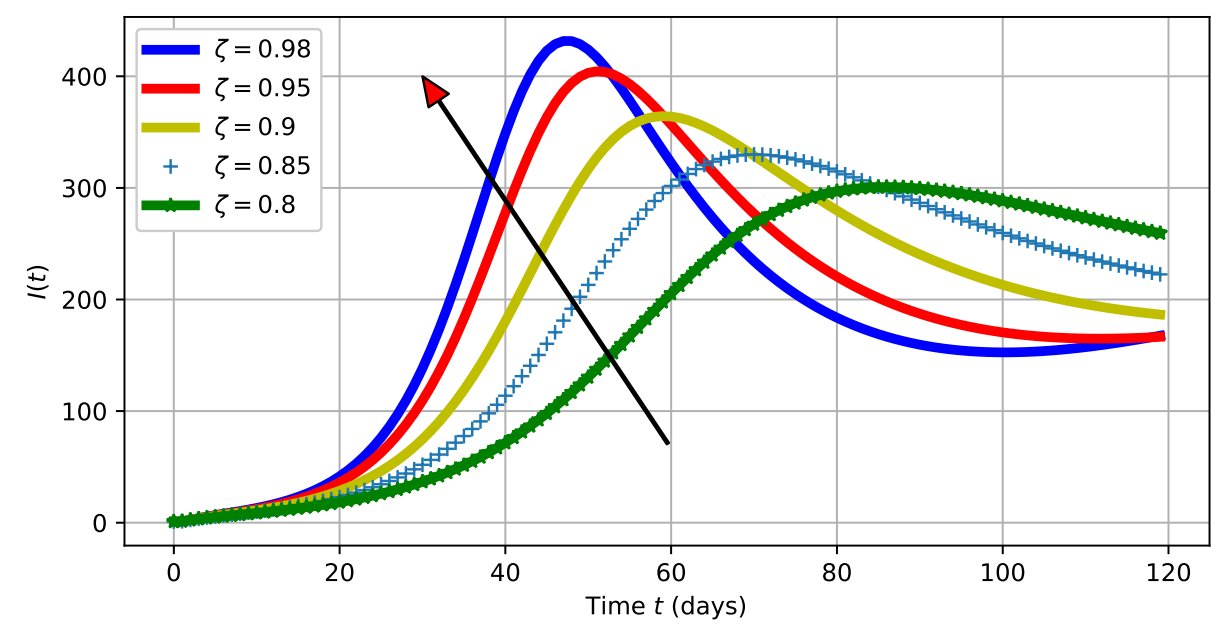

Fig. 7. Evolution of the infected compartment for varying fractional order $\zeta$.

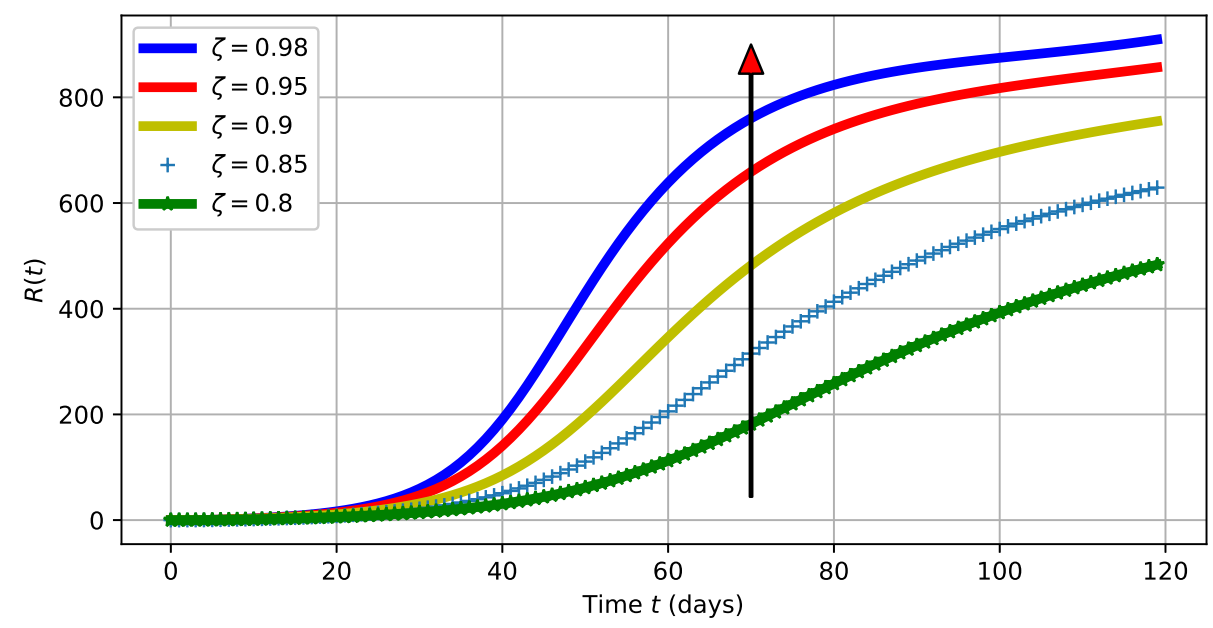

Fig. 8. Evolution of the recovered compartment for varying fractional order $\zeta$.

predict the worst cases and guide public health actions to achieve the best possible result. The precision of the model predictions turns entirely on the quantity/quality of epidemiological data used and on the understanding of the transmission dynamics of the disease. Both elements are expected to improve and refine over time the model. We believe that the model of this paper makes an attempt of the disease dynamics, so, we will focus in future on a comprehensible depiction of COVID-19 in Morocco with a complete data record and under different intervention strategies.

\section{ACKNOWLEDGEMENTS}

H. A. and M. T. are grateful to the support of the Moroccan MENFPESRS /CNRST through the Scientific and Technological Research Support Program "Analyse épidémique du COVID-19 au Maroc par modélisation dynamique et intelligence artificielle”. 


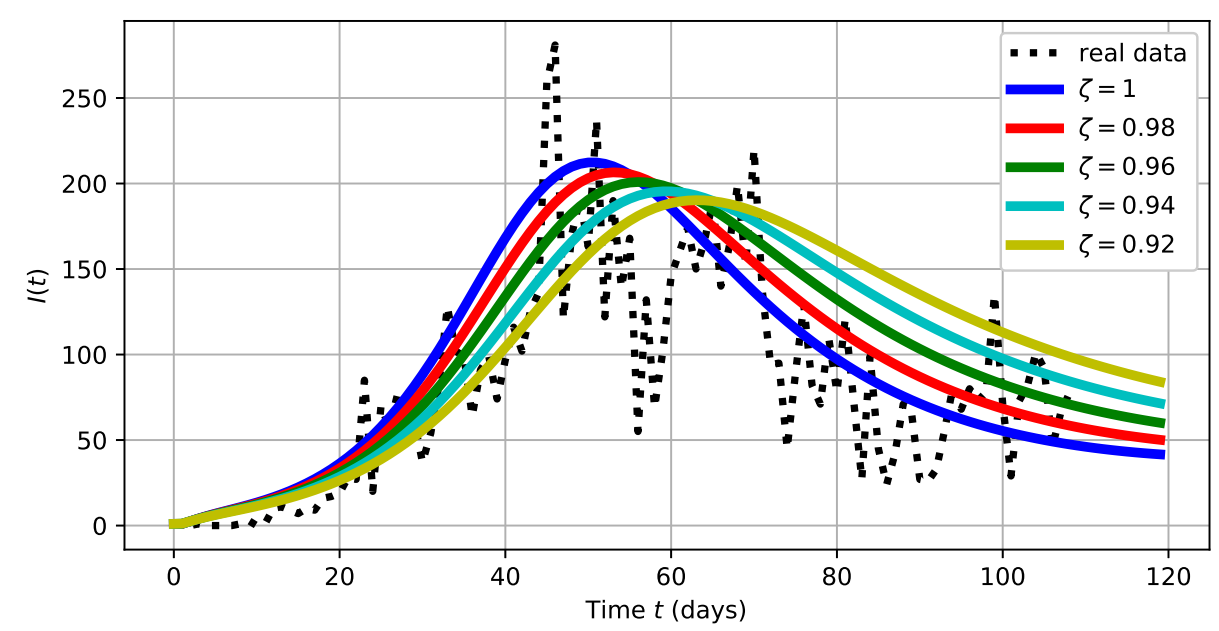

Fig. 9. Infected population for different values of $\zeta$ with $S_{0}=798, E_{0}=5, I_{0}=1, R_{0}=0$, $\mu=10^{-2}$ and $\Lambda=10$.

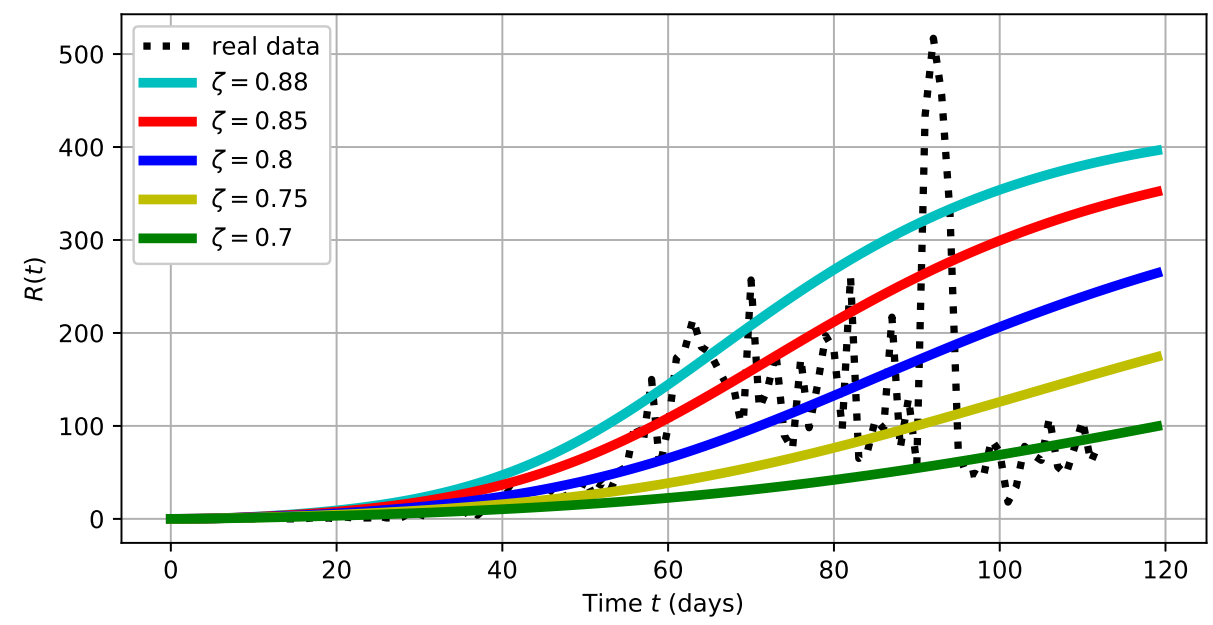

Fig. 10. Recovered population with $S_{0}=798, E_{0}=5, I_{0}=1, R_{0}=0, \mu=10^{-2}$ and $\Lambda=10$.

\section{REFERENCES}

1. Atangana A. Modelling the spread of COVID-19 with new fractal-fractional operators: Can the lockdown save mankind before vaccination? Chaos, Solitons \& Fractals. 2020. V. 136. P. 109860. doi: 10.1016/j.chaos.2020.109860.

2. Atangana A., Araz S.I. Mathematical model of COVID-19 spread in Turkey and South Africa: Theory, methods and applications. Advances in Difference Equations. 2020. V. 2020. №1. P. 1-89. doi: 10.1101/2020.05.08.20095588

3. Yang Y., Lu Q., Liu M., Wang Y., Zhang A., Jalali N., Dean N.E., Longini I., Halloran M.E., $\mathrm{Xu}$ B., et al. Epidemiological and clinical features of the 2019 novel coronavirus outbreak in China. medRxiv. 2020. V. 2020. №1. P. 1-89. doi: $10.1101 / 2020.02 .10 .20021675$

4. Diekmann O., Heesterbeek J.A.P., Metz J.A.J. On the definition and the computation of the basic reproduction ratio R0 in models for infectious diseases in heterogeneous populations. Journal of Mathematical Biology. 1990. V. 28. №4. P. 365-382. doi: 10.1007/BF00178324 
5. World Health Organization Website. URL: https://www.who.int/ (accessed 29.04.2021).

6. Atangana A., Alkahtani B.S. Analysis of the Keller-Segel model with a fractional derivative without singular kernel. Entropy. 2015. V. 17. №6. P. 4439-4453. doi: 10.3390/e17064439

7. Atangana A., Baleanu D. New fractional derivatives with nonlocal and $\overline{\text { non-singular kernel: }}$ Theory and application to heat transfer model. Thermal Science. 2016. V. 20. P. 763-769. doi: $10.2298 /$ TSCI160111018A

8. Atangana A., Koca I. On the new fractional derivative and application to nonlinear Baggs and Freedman model. J. Nonlinear Sci. Appl. 2016. V. 9. №5. P. 2467-2480. doi: 10.22436/jnsa.009.05.46

9. Yavuz M., Özdemir N., Baskonus H.M. Solutions of partial differential equations using the fractional operator involving Mittag-Leffler kernel. The European Physical Journal Plus. 2018. V. 133. №6. P. 1-11. doi: 10.1140/epjp/i2018-12051-9

10. Saad K.M., Deniz S., Baleanu D. On a new modified fractional analysis of Nagumo equation. International Journal of Biomathematics. 2019. V. 12. №03. P. 1950034. doi: $\underline{10.1142 / \mathrm{S} 1793524519500347}$

11. Yusuf A., Qureshi S., Inc M., Aliyu A.I., Baleanu B., Shaikh A.A. Two-strain epidemic model involving fractional derivative with Mittag-Leffler kernel. Chaos: An Interdisciplinary Journal of Nonlinear Science. 2018. V. 28. №12. P. 123121. doi: $\underline{10.1063 / 1.5074084}$

12. Bildik N., Deniz S. A new fractional analysis on the polluted lakes system. Chaos, Solitons \& Fractals. 2019. V. 122. P. 17-24. doi: 10.1016/j.chaos.2019.02.001

13. Uçar S. Existence and uniqueness results for a smoking model with determination and education in the frame of non-singular derivatives. Discrete Contin. Dyn. Syst. Ser. A. 2018. P. 1-17. doi: $10.3934 /$ dcdss. 2020178

14. Hethcote H. The mathematics of infectious diseases. SIAM Review. 2000. V. 42. №4. P. 599-653. doi: $10.1137 / \mathrm{S} 0036144500371907$

15. Brauer F. Mathematical epidemiology: Past, present, and future. Infectious Disease Modelling. 2017. V. 2. №2. P. 113-127. doi: 10.1016/j.idm.2017.02.001

16. Yang C., Wang J. A mathematical model for the novel coronavirus epidemic in Wuhan, China. Mathematical Biosciences and Engineering. 2020. V. 17. №3. P. 2708. doi: $10.3934 / \mathrm{mbe} .2020148$

17. Khan M.A., Ullah S., Farooq M. A new fractional model for tuberculosis with relapse via Atangana-Baleanu derivative. Chaos, Solitons \& Fractals. 2018. V. 116. P. 227-238. doi: 10.1016/j.chaos.2018.09.039

18. Khan M.A., Atangana A. Modeling the dynamics of novel coronavirus (2019-nCov) with fractional derivative. Alexandria Engineering Journal. 2020. V. 59. №4. P. 2379-2389. doi: 10.1016/j.aej.2020.02.033

19. Ullah M.Z., Alzahrani A.K., Baleanu D. An efficient numerical technique for a new fractional tuberculosis model with nonsingular derivative operator. Journal of Taibah University for Science. 2019. V. 13. №1. P. 1147-1157. doi: $\frac{10.1080 / 16583655.2019 .1688543}{1}$

20. Khan M.A., Atangana A. Dynamics of Ebola Disease in the Framework of Different Fractional Derivatives. Entropy. 2019. V. 21. №3. P. 303. doi: 10.3390/e21030303

21. Khan M.A., Ismail M., Ullah S., Farhan M. Fractional order SIR model with generalized incidence rate. AIMS Mathematics. 2020. V. 5. №3. P. 1856-1880. doi: $10.3934 /$ math.2020124

22. The Moroccan Ministry of Public Health, COVID-19 Platform. URL: http://www.covidmaroc.ma/Pages/AccueilAR.aspx (accessed 29.04.2021).

23. Shaikh A.S., Shaikh I.N., Nisar K.S. A Mathematical Model of COVID-19 Using Fractional 
Derivative: Outbreak in India with Dynamics of Transmission and Control. Advances in Difference Equations. 2020. V. 2020. №1. P. 1-19. doi: 10.20944/preprints202004.0140.v1.

24. Shaikh A.S., Jadhav V.S., Timol M.G., Nisar K.S., Khan I. Analysis of the COVID-19 Pandemic Spreading in India by an Epidemiological Model and Fractional Differential Operator. 2020. Preprints. P. 1-16. doi: 10.20944/preprints202005.0266.v1.

25. Kermack W.O., McKendrick A.G. A contribution to the mathematical theory of epidemics. Proceedings of the Royal Society of London. Series A, Containing Papers of a Mathematical and Physical haracter. 1927. V. 115. №772. P. 700-721. doi: 10.1098/rspa.1927.0118

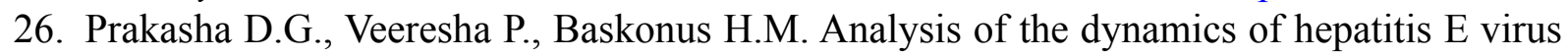
using the Atangana-Baleanu fractional derivative. The European Physical Journal Plus. 2019. V. 134. №5. P. 1-11. doi: 10.1140/epjp/i2019-12590-5

27. Mekkaoui T., Atangana A. New numerical approximation of fractional derivative with non-local and non-singular kernel: application to chaotic models. The European Physical Journal Plus. 2017. V. 132. №10. P. 1-16. doi: 10.1140/epjp/i2017-11717-0

28. Atangana A., Owolabi K.M., New numerical approach for fractional differential equations. Mathematical Modelling of Natural Phenomena. 2018. V. 13. №1. P. 3. doi: $10.1051 / \mathrm{mmnp} / 2018010$

29. Rihan F.A., Al-Mdallal Q.M., AlSakaji H.J., Hashish A. A fractional-order epidemic model with time-delay and nonlinear incidence rate. Chaos, Solitons \& Fractals. 2019. V. 126. P. 97-105. doi: 10.1016/j.chaos.2019.05.039

30. Ahmad S., Ullah A., Al-Mdallal Q.M., Khan H., Shah K., Khan A. Fractional order mathematical modeling of COVID-19 transmission. Chaos, Solitons \& Fractals. 2020. V. 139. P. 110256. doi: $10.1016 /$ j.chaos.2020.110256

31. Asif M., Khan Z.A., Haider N., Al-Mdallal Q., Numerical simulation for solution of SEIR models by meshless and finite difference methods. Chaos, Solitons \& Fractals. 2020. V. 141. P. 110340. doi: 10.1016/j.chaos.2020.110340

32. Asif M., Jan S.U., Haider N., Al-Mdallal Q., Abdeljawad T. Numerical modeling of NPZ and SIR models with and without diffusion. Results in Physics. 2020. V. 19. P. 103512. doi: 10.1016/j.rinp.2020.103512

33. Hajji M.A., Al-Mdallal Q. Numerical simulations of a delay model for immune system-tumor interaction. Sultan Qaboos University Journal for Science. 2018. V. 23. №1. P. 19-31. doi: 10.24200/squjs.vol23iss1pp19-31

34. Thabet S.T.M., Abdo M.S., Shah K., Abdeljawad T. Study of transmission dynamics of COVID-19 mathematical model under ABC fractional order derivative. Results in Physics. 2020. V. 19. P. 103507. doi: $\underline{10.1016 / \text { j.rinp.2020.103507 }}$

Accepted 19.02.2021.

Revised 18.04.2021.

Published 08.05.2021. 